\title{
Commentary on the Paper "Anatomical Study of the Caudate Lobe with Special Reference to the Portal Venous and Biliary Branches Using Corrosion Liver Casts and Clinical Application" by Kumon
}

\author{
Guido Torzilli \\ School of Medicine, Humanitas University, Humanitas Research Hospital, IRCCS, Rozzano, Italy
}

This translation paper describes the anatomy of the caudate lobe using corrosion liver casts. It was originally published in Japanese more than 30 years ago [1]. Dr. Kumon studied 23 elaborative corrosion liver casts of autopsied livers. The corrosion liver casts were prepared by injecting colored silicone rubber into hepatic vessels and removing the hepatic parenchyma. Through the analysis, Dr. Kumon proposed that the caudate lobe can be partitioned into 3 regions by focusing on the type of the portal venous and biliary branches that are distributed in the caudate lobe: the Spiegel lobe, paracaval portion, and caudate process portion. In Europe, the anatomical concept of the caudate lobe was based on Couinaud's analysis [2]: he divided the caudate lobe into the left (segment 1l) and right (segment 1r) segments, although this definition had been slightly modified according to the period. In the US, Healey and Schroy [3] reported that the caudate lobe was partitioned into 3 portions that comprise the left portion, right portion, and caudate process.

Finely prepared corrosion liver casts successfully demonstrated portal venous branches that ramified from the right and left portal veins (Fig. 1) and the extent of the caudate lobe (Fig. 2, 4). Schematic images in Figure 4 depicted the anatomical concept of the caudate lobe by Kumon and colleagues, which comprised the Spiegel lobe, paracaval portion, and caudate process portion. This partition system influenced particularly the hepatobiliary teams in Japan in the 1990s and 2000s, but now is considered also by the European groups. In the event of surgery for hepatocellular carcinoma, the anatomic resection of Couinaud segment 1 , using the counterstaining [4] or the counter-compression technique [5], has been possible. Similarly, right trisectionectomy with full resection of segment 1 for hilar-cholangiocellular carcinoma [6] and left liver procurement including segment 1 for donor surgery [7] have also been described. Conservative surgery for anatomically removed large tumors in segment 1 has also been supported [8]. Based on this partition system, and on the evidence that, in the case of colorectal liver metastases, the tumor-vessel detachment is suitable [9], the liver 
Torzilli: Commentary

tunnel has recently been proposed [10], including full removal of segments 1 and 8 , with or without the middle hepatic vein and the segment 4 superior.

Although the original article was published more than 30 years ago, I believe it is still informative for today's liver surgeons and increases the understanding of the anatomy and the surgical management of the caudate lobe. The recent literature, also released by European groups of which the author is representative, is a practical example of the central role played by the study of Dr. Kumon: the entire community of hepatobiliary surgeons should be grateful to him.

I also congratulate Prof. Kokudo, Dr. Sakamoto, Dr. Kawaguchi, and Dr. Maki for the second publication project to internationally introduce Dr. Kumon's work.

\section{References}

1 Kumon M: Portal vein and bile duct branches of the caudate lobe analysis of liver cast and clinical cases (in Japanese). Kanzou (Acta Hepatol Jpn) 1985;26:1193-1199.

2 Couinaud C: Surgical Anatomy of the Liver Revisited. Paris, C Couinaud, 1989.

3 Healey JE Jr, Schroy PC: Anatomy of the biliary ducts within the human liver; analysis of the prevailing pattern of branchings and the major variations of the biliary ducts. AMA Arch Surg 1953;66:599-616.

4 Takayama T, Makuuchi M, Watanabe K, Kosuge T, Takayasu K, Yamazaki S, Hasegawa H: A new method for mapping hepatic subsegment: counterstaining identification technique. Surgery 1991;109:226-229.

5 Torzilli G, Procopio F, Cimino M, Del Fabbro D, Palmisano A, Donadon M, Montorsi M: Anatomical segmental and subsegmental resection of the liver for hepatocellular carcinoma: a new approach by means of ultrasound-guided vessel compression. Ann Surg 2010;251:229-235.

6 Nagino M, Kamiya J, Arai T, et al: "Anatomic" right hepatic trisectionectomy (extended right hepatectomy) with caudate lobectomy for hilar cholangiocarcinoma. Ann Surg 2006;243:28-32.

7 Takayama T, Makuuchi M, Kubota K, et al: Living-related transplantation of left liver plus caudate lobe. J Am Coll Surg 2000;190:635-638.

8 Viganò L, Costa G, Procopio F, Donadon M, Cimino M, Del Fabbro D, Gatti A, Torzilli G: Parenchyma-sparing liver surgery for large segment 1 tumors: ultrasound-guided lateral and superior approaches as safe alternatives to major hepatectomy. J Am Coll Surg 2015;221:e65-e73.

9 Viganò L, Procopio F, Cimino MM, Donadon M, Gatti A, Costa G, Del Fabbro D, Torzilli G: Is tumor detachment from vascular structures equivalent to r0 resection in surgery for colorectal liver metastases? An observational cohort. Ann Surg Oncol 2016;23:1352-1360.

10 Torzilli G, Cimino M, Procopio F, Costa G, Donadon M, Del Fabbro D, Gatti A, Garcia-Etienne CA: Conservative hepatectomy for tumors involving the middle hepatic vein and segment 1: the liver tunnel. Ann Surg Oncol 2014;21:2699. 